\title{
PREFERENCIAS POR LAS DECISIONES COMPARTIDAS EN PACIENTES CON DEPRESIÓN
}

Ascensión Fumero Hernández*, Rosario J. Marrero Quevedo, Carlos de las Cuevas Castresana, Wenceslao Peñate Castro

Facultad de Ciencias de la Salud - Universidad de La Laguna - España

Recibido, septiembre $11 / 2015$

Concepto evaluación, noviembre 4/2015

Aceptado, noviembre 20/2015
Referencia: Fumero Hernández, A., Marrero Quevedo, R.J., De las Cuevas Castresana, C. \& Peñate Castro, W. Preferencias por las decisiones compartidas en pacientes con depresión. Acta Colombiana de Psicología, 19(1), 249-259. DOI: 10.14718/ACP.2016.19.1.11

Resumen

En el presente estudio se analizaron los procesos psicológicos asociados con las preferencias de los pacientes con depresión en la toma de decisión sobre su tratamiento psiquiátrico. Participaron 462 pacientes diagnosticados con un trastorno depresivo agudo o recurrente. La mayor parte prefirió asumir un rol colaborativo-pasivo o totalmente pasivo. Los resultados no mostraron diferencias significativas entre pacientes en función de su cronicidad en la preferencia por la toma de decisiones, aunque un mayor tiempo de tratamiento se asoció con un estilo más pasivo. El MANCOVA aplicado al total de participantes, controlando la edad, el nivel educativo y el tiempo de tratamiento, indicó que los pacientes colaborativos y pasivos mostraron mayor locus de control centrado en la confianza en el psiquiatra que los activos. Se encontraron diferencias de género mostrando en los hombres mayor locus de control interno y reactancia psicológica, y en las mujeres, mayor locus centrado en el azar. Los análisis de regresión indicaron que en el caso de los hombres, la preferencia pasiva por la toma de decisión es explicada por el locus centrado en el psiquiatra. Sin embargo, en las mujeres tuvo mayor peso la edad, seguida del locus centrado en el azar, el locus centrado en el psiquiatra y una percepción de menor autoeficacia. Los hallazgos señalan la necesidad de estudiar desde una perspectiva diferencial la participación de los pacientes en la toma de decisión de acuerdo con los procesos psicológicos, así como la repercusión que esta tiene en la adherencia al tratamiento médico.

Palabras clave: toma de decisión compartida, locus de control, reactancia psicológica, autoeficacia, depresión.

\section{DEPRESSIVE PATIENTS' PREFERENCES IN SHARED DECISION-MAKING}

\begin{abstract}
This study analyzed the role of psychological processes predicting depressed patients' preferences in clinical decision-making about psychiatric treatment. 462 patients diagnosed with depressive disorders, acute or recurrent, participated in a crosssectional survey. Most participants preferred collaborative-passive or totally passive roles. Results showed no significant differences between acute and recurrent patients in their preference of participation in decision-making, but longer treatment duration was associated with a more passive style. MANCOVA, controlling age, educational level and treatment duration variables, showed that collaborative and passive patients had a greater locus of control focused on their psychiatrist than active patients. Gender differences were found. Men showed greater internal locus of control and psychological reactance, while women showed greater external locus of control focused on chance. Regression analysis indicated that, for men, passive preferred role was explained by external locus centered on their psychiatrist. However, age registered the highest weight for women' passive decision-making, followed by the locus focused on chance, locus focused on the psychiatrist and lower self-efficacy. Our findings suggest the need to study shared decision-making from a differential perspective that involves psychological processes and the impact of these processes in adherence to medical treatments.

Key words: shared decision-making, health locus of control, psychological reactance, self-efficacy, depression.
\end{abstract}

* Ascensión Fumero, Departamento de Psicología Clínica, Psicobiología y Metodología, Facultad de Ciencias de la Salud- Sección Psicología. Campus de Guajara, Universidad de La Laguna 38200 Tenerife (Spain). Tel: 34-922317960. afumero@ull.es

Este trabajo se ha desarrollado con el apoyo del Instituto de Salud Carlos III, FEDER Unión Europea (PI10/00955) 


\title{
PREFERÊNCIAS PELAS DECISÓES COMPARTILHADAS EM PACIENTES COM DEPRESSÃO
}

\begin{abstract}
Resumo
Neste estudo, analisaram-se os processos psicológicos associados com as preferências dos pacientes com depressão na tomada de decisão sobre seu tratamento psiquiátrico. Participaram 462 pacientes diagnosticados com um transtorno depressivo agudo ou recorrente. A maior parte preferiu assumir um papel colaborativo-passivo ou totalmente passivo. Os resultados não mostraram diferenças significativas entre pacientes em função de sua cronicidade na preferência por tomada de decisões, embora um maior tempo de tratamento tenha sido associado com um estilo mais passivo. $\mathrm{O}$ MANCOVA aplicado ao total de participantes, controlando a idade, o nível educativo e o tempo de tratamento, indicou que os pacientes colaborativos e passivos mostraram maior lócus de controle centralizado na confiança no psiquiatra do que os ativos. Constataram-se diferenças de gênero que mostraram nos homens maior lócus de controle interno e reatância psicológica, e, nas mulheres, maior lócus centralizado no aleatório, o lócus centralizado no psiquiatra e uma percepção de menor autoeficácia. Os achados indicam a necessidade de estudar, sob uma perspectiva diferencial, a participação dos pacientes na tomada de decisão de acordo com os processos psicológicos e a repercussão que esta tem na aderência ao tratamento médico.

Palavras-chave: tomada de decisão compartilhada, lócus de controle, reatância psicológica, autoeficácia, depressão.
\end{abstract}

\section{INTRODUCCIÓN}

Los trastornos del estado de ánimo han sido uno de los problemas de salud pública más relevantes en los últimos tiempos. En España, entre 2006 y 2010, en los servicios de Atención Primaria, se incrementó la prevalencia de la depresión mayor en un $19.4 \%$ y la de episodios depresivos en un 4.7\% (Gili, Roca, Basu, McKee, \& Stuckler, 2013). La depresión lleva aparejadas diversas complicaciones médicas, un gran deterioro funcional y, en muchas ocasiones, una escasa adherencia a los regímenes de tratamiento y de cuidado personal que puede repercutir en un mayor riesgo de morbilidad y mortalidad (Katon, 2011).

En la práctica clínica, la falta de adherencia a la medicación antidepresiva es una barrera importante para el éxito del tratamiento de la depresión(De las Cuevas, \& Peñate, 2014; Grenard et al., 2011; Pérez-Wehbe, Perestelo, Bethencourt, Cuéllar, \& Peñate, 2014). La literatura ha mostrado que la falta de adherencia se acompaña de peores resultados del tratamiento, mayor riesgo de recaídas y recurrencia, además de suponer un aumento de los costes sanitarios (Cantrell, Eaddy, Shah, Regan, \& Sokol, 2006; Geddes et al., 2003). Estos efectos tienen mayor repercusión en los pacientes de larga duración que requieren una mayor constancia en la utilización de medicación (Katon., 2011) y en los que la falta de adherencia va a incrementar la cronicidad del trastorno.

Se ha sugerido que uno de los factores que podría mejorar la adherencia al tratamiento y la eficacia del mismo es la toma de decisión compartida (SDM, por sus siglas en inglés). En ella, el profesional sanitario intercambia información con el paciente sobre el mejor tratamiento disponible y se discuten las implicaciones de cada opción (Makoul \& Clayman, 2006). Los pacientes pueden participar de la búsqueda e intercambio de información, el debate sobre opciones en el cuidado y la toma de decisión sobre el tratamiento. La toma de decisión compartida ha mostrado ser eficaz en enfermedades crónicas y principalmente cuando los pacientes necesitan diversas sesiones de intervención (Joosten et al., 2008). De ahí la importancia en la atención a los trastornos en salud mental. Sin embargo, no todos los pacientes están preparados o quieren participar en el mismo grado en el proceso de toma de decisión sobre el tratamiento de su problema. Los estudios muestran que aunque algunos pacientes desean jugar un papel activo en la discusión de las opciones de tratamiento, en última instancia quieren que sus médicos tomen la decisión en su nombre (Levinson, Kao, Kuby, \& Thisted, 2005).

La adecuación de una decisión en SDM depende del contexto clínico, de la responsabilidad de los profesionales de la salud, de las preferencias del paciente y de ciertas características sociodemográficas (Longtin et al., 2010). Se ha encontrado que aunque mujeres y hombres no se diferencian en preferencias, las mujeres asumen un papel más pasivo que los hombres. Del mismo modo, las personas de más edad y menor nivel educativo han mostrado implicarse en menor medida en la toma de decisión, mostrando 
estilos más pasivos o paternalistas (Schneider et al., 2006; Singh et al., 2010).

En pacientes depresivos, tanto la toma de decisión compartida como la atención recibida por el servicio de salud mental se ha asociado con mayor satisfacción del paciente (Aljumah, \& Hassali, 2015; Swanson, Bastani, Rubenstein, Meredith, \& Ford, 2007). Además, cuando el personal médico es entrenado para llevar a cabo la toma de decisión compartida, se encuentra que hay una mayor implicación del paciente en el tratamiento (Loh et al., 2007).

Asimismo, la reactancia psicológica, el locus de control relacionado con la salud y el sentido de autoeficacia han mostrado ser procesos psicológicos que pueden estar incidiendo en el grado de implicación del paciente en las distintas cuestiones relativas a su salud. La reactancia psicológica es una reacción afectiva aversiva en respuesta a las imposiciones que inciden sobre la autonomía del individuo (Brehm \& Brehm, 2013). Las recomendaciones para seguir las prescripciones médicas tienen el potencial para provocar reactancia y, como resultado, llevar a los pacientes a ignorar el tratamiento recomendado (De Almeida \& Chen, 2008). La reactancia psicológica ha mostrado estar relacionada negativamente con la adherencia al tratamiento en pacientes con trastorno depresivo (De las Cuevas, Peñate, \& Sanz, 2014). Del mismo modo, la reactancia tendrá repercusiones en el grado en que la persona va a participar en la toma de decisiones médicas.

Por su parte, el locus de control relacionado con la salud se refiere a las creencias del individuo sobre las causas y el resultado del comportamiento (Wallston, 1992). Un paciente con un locus de control interno cree que la salud es el resultado directo de su propio comportamiento, mientras que un paciente con un locus externo cree que la salud es consecuencia de la casualidad o de la intervención de otras personas, como profesionales de la medicina. El locus de control de salud se ha relacionado de manera fiable con los resultados médicos (O'hea et al., 2005) y con la preferencia de los pacientes por obtener información e implicarse en las decisiones médicas. Las personas con puntuaciones altas en depresión tienen menor preferencia por la información y mayor locus de control fatalista (Schneider et al., 2006). Además, a mayor edad, menor implicación en la toma de decisión. En el caso de las mujeres se han encontrado mayores niveles de creencias en el control de la salud que en los hombres (Pudrovska, 2015).

Por otro lado, el sentido de autoeficacia refleja la creencia en las propias habilidades para planificar y llevar a cabo actividades que favorezcan el hecho de alcanzar objetivos particulares (Bandura, 1977; Schwarzer \& Fuchs, 1996), produciendo un mayor sentido de confianza y control, que se traduce en un incremento en la probabilidad de realizar un comportamiento. La autoeficacia representa uno de los factores protectores de la depresión y se ha asociado con una mayor calidad de vida en pacientes depresivos (Botero \& Londoño, 2013; Serra-Taylor \& Irizarry-Robles, 2015). Las personas con mayor confianza en sus habilidades para seguir un plan de tratamiento y lograr el resultado deseado se implican más en las conductas necesarias (Makoul \& Clayman, 2006).

Con base en los estudios previos, el objetivo de este trabajo ha sido analizar el papel de los procesos psicológicos (locus de control, reactancia psicológica y autoeficacia) que pueden determinar las preferencias de los pacientes depresivos en la toma de decisiones médicas. Además, se ha examinado la capacidad predictiva de estos procesos psicológicos frente a características sociodemográficas y clínicas. Específicamente, los objetivos del presente trabajo son los siguientes: a) Conocer las relaciones entre las variables sociodemográficas y clínicas con las preferencias en la toma de decisión, en pacientes con depresión; b) Analizar la relación entre procesos psicológicos de locus de control, reactancia psicológica y autoeficacia en la preferencia por una toma de decisión determinada; c) Estudiar si las mujeres con trastorno depresivo presentan un tipo de preferencia diferente al de los hombres con depresión; d) Identificar si los procesos psicológicos explican en mayor medida las preferencias en la toma de decisión que las características sociodemográficas y/o clínicas.

\section{MÉTODO}

\section{Participantes}

En este estudio participaron 462 pacientes del Servicio de Salud Mental del Hospital Nuestra Señora de la Candelaria de Tenerife. El criterio de inclusión fue haber sido diagnosticados con algún trastorno depresivo (F32 o F33), según la International Classification of Diseases-ICD-10 (WHO, 2004). Todos los participantes fueron informados del objetivo general del estudio y se les solicitó el consentimiento informado para emplear los datos recabados con fines de investigación. El marco temporal del estudio fue de octubre de 2012 a abril de 2014.

En la tabla 1 se presentan las características sociodemográficas y clínicas de los participantes diagnosticados con trastorno depresivo. El rango de edad osciló entre los 18 y 85 años, aunque la mayor proporción estuvo entre los $45 \mathrm{y}$ 60 años. Más de tres cuartas partes de los participantes eran mujeres. Había una proporción similar de participantes en los distintos niveles académicos, sólo un $10.6 \%$ no había finalizado sus estudios. La mayor parte de ellos recibían 
medicación para controlar el trastorno y llevaban en promedio siete años con el tratamiento. Aproximadamente la mitad tenía un episodio depresivo, y el resto trastorno depresivo recurrente. En general, los participantes preferían asumir un rol colaborativo-pasivo o completamente pasivo en relación con la toma de decisión compartida.

Tabla 1.

Características sociodemográficas y clínicas de los participantes $(\mathrm{N}=466)$

\begin{tabular}{|c|c|c|c|}
\hline Variables & Categorías & $\mathrm{N}$ & Porcentaje \\
\hline \multirow[t]{5}{*}{$\operatorname{Edad}(\bar{X}=54.29 \pm 13.3)$} & $18-30$ & 25 & 5.4 \\
\hline & $30-45$ & 76 & 16.5 \\
\hline & $45-60$ & 215 & 46.6 \\
\hline & $60-75$ & 123 & 26.7 \\
\hline & $>75$ & 22 & 4.8 \\
\hline \multirow[t]{2}{*}{ Género } & Hombres & 98 & 21.3 \\
\hline & Mujeres & 363 & 78.7 \\
\hline \multirow[t]{4}{*}{ Nivel educativo } & Sólo leer y escribir & 49 & 10.6 \\
\hline & Primarios & 165 & 35.7 \\
\hline & Secundarios & 150 & 32.5 \\
\hline & Universitarios & 98 & 21.2 \\
\hline \multirow[t]{2}{*}{ Diagnóstico } & Episodio depresivo & 237 & 51.3 \\
\hline & Trastorno depresivo recurrente & 225 & 48.7 \\
\hline \multicolumn{4}{|c|}{ Tiempo de tratamiento $(\bar{X}=93.60$ meses \pm 97.55$)$} \\
\hline \multirow[t]{6}{*}{ Número de medicamentos } & Ninguno & 8 & 1.7 \\
\hline & Uno & 54 & 11.8 \\
\hline & Dos & 142 & 30.9 \\
\hline & Tres & 132 & 28.8 \\
\hline & Cuatro & 74 & 16.1 \\
\hline & Cinco o más & 49 & 10.7 \\
\hline \multicolumn{4}{|c|}{ Preferencia en toma de decisiones } \\
\hline \multirow[t]{2}{*}{ Activo } & Activo-activo & 3 & .6 \\
\hline & Activo-colaborativo & 18 & 3.9 \\
\hline \multirow[t]{2}{*}{ Colaborativo } & Colaborativo-activo & 10 & 2.2 \\
\hline & Colaborativo-pasivo & 294 & 63.6 \\
\hline \multirow[t]{2}{*}{ Pasivo } & Pasivo-colaborativo & 22 & 4.8 \\
\hline & Pasivo-pasivo & 115 & 24.9 \\
\hline
\end{tabular}




\section{Instrumentos}

Las características sociodemográficas y clínicas se evaluaron mediante una entrevista semiestructurada y a través de la historia clínica de los participantes. Entre las características sociodemográficas se registró la edad, el género y el nivel de estudios (sin estudios, estudios primarios, estudios secundarios y estudios universitarios). Entre las características clínicas se consideró el diagnóstico, identificando a los individuos con episodio depresivo o trastorno depresivo recurrente, con base en la ICD-10. También se registraron los meses que llevaban con el tratamiento farmacológico y la cantidad de fármacos que tenían prescritos (ninguno, uno, dos, tres, cuatro y cinco o más psicotrópicos).

La Escala de Preferencias de Control-CPS [por sus siglas en inglés] (Degner, Sloan, \& Venkatesh, 1997) fue diseñada para evaluar el grado de control que los pacientes asumen cuando deben tomar decisiones acerca del tratamiento médico. A los individuos se les presentaban cinco tarjetas en las que aparecía representada la interacción de médico y paciente, así como una frase que reflejaba quién tenía el papel más activo en la decisión. Los pacientes debían elegir entre las tarjetas, observándolas una por una, para establecer un orden de preferencia, desde un papel totalmente activo hasta un estilo más pasivo (de 0 a 5 , donde la mayor puntuación correspondía a un estilo más pasivo). Los participantes respondían a la prueba en dos ocasiones, antes de entrar a la consulta y al finalizar la misma; de este modo se analizó la variable de forma continua. También se analizó desde un punto de vista categorial, la toma de decisión preferida, la cual estaba dividida en tres estilos, dando prioridad a la tarjeta que los participantes escogían por primera vez. Estos estilos eran: a) activo, el cual incluía a las personas que en ambos momentos de la prueba escogían un estilo activo, y las que escogían primero el activo y luego el colaborativo; b) colaborativo, que incluía a quienes elegían este estilo en primer lugar, independientemente de que luego escogieran un estilo activo o pasivo; c) pasivo, que se refería a las personas que preferían un estilo pasivo y luego uno colaborativo o los que escogían en las dos ocasiones un estilo pasivo. Al respecto, los estudios realizados por Tariman, Doorenbos, Schepp, Singhal, \& Berry (2014) dan cuenta de la estabilidad de la preferencia en la toma de decisión en un $50 \%$ de los participantes.

La Escala Multidimensional de Locus de Control de la Salud-MILC- Forma C (Wallston, Stein, \& Smith, 1994). Este instrumento está compuesto por 18 ítems que evalúan el locus de control del individuo en el contexto de la salud. Se emplea una escala de respuesta tipo Likert de seis puntos que oscila desde el desacuerdo hasta el total acuerdo, de modo que una alta puntuación supone un mayor locus de control. La prueba permite obtener cuatro dimensiones: locus de control interno, que refleja el grado en que el individuo considera que su salud depende de su comportamiento; locus de control centrado en el azar, que se refiere a la creencia en que la salud depende de la suerte, del azar o el destino; locus de control centrado en el profesional sanitario, que muestra la creencia de que esta es la persona que determina el estado de salud del paciente; y locus de control centrado en otras personas, que consiste en situar en la familia o en los amigos el control del estado de salud. Se empleó la versión validada en español de la escala, la cual ha mostrado un $\alpha$ de Cronbach aceptable: .74 para locus interno, .65 locus-azar, .54 locus-médico y .48 locus-otras personas (De las Cuevas, Peñate, Betancort, \& Cabrera, 2015).

La Escala de Autoeficacia General-GSS-(Jerusalem \& Schwarzer, 1992). Está formada por diez ítems que miden en una escala de 1 (totalmente falso) a 4 (totalmente cierto) la creencia en que las acciones del individuo inciden en el manejo exitoso de las situaciones. A mayor puntuación en la escala, mayor autoeficacia. En este estudio se ha utilizado la versión de la escala validada al español que cuenta con un $\alpha$ de Cronbach de 90 (Sanjuán, Pérez, \& Bermúdez, 2000).

La Escala de Reactancia Psicológica de Hong-HPRS(Hong \& Faedda, 1996). Está formada por 14 ítems que miden la reactancia cognitiva y afectiva, a través de una escala de tipo Likert que oscila entre 1 (total desacuerdo) y 5 (total acuerdo). La reactancia sucede cuando se amenaza la libertad del individuo de modo que se produce una reacción para recuperar dicha libertad perdida (Wallston, 1992). La escala ha demostrado tener una adecuada consistencia interna de .76 para reactancia afectiva y .62 para reactancia cognitiva (De las Cuevas, Peñate, Betancort, \& de Rivera, 2014).

\section{Procedimiento}

Se accedió a los participantes a través de los profesionales del Servicio de Salud Mental del Hospital Nuestra Señora de la Candelaria que fueron los encargados de informar a los pacientes de la investigación. Tras la firma del consentimiento informado se registraban distintas características sociodemográficas y clínicas y los pacientes recibían un protocolo de evaluación que contestaban en la sala de espera de su Unidad de Salud Mental con una duración aproximada de 35 minutos. Análisis de datos

Para el análisis de datos se empleó el programa estadístico SPSS versión 21. Se analizó la distribución de las distintas características sociodemográficas y clínicas registradas. Posteriormente, se comprobó si existían diferencias en las distintas características registradas entre los pacientes, según estuvieran diagnosticados como episodio depresivo o trastorno depresivo recurrente, a través de la prueba de $\chi^{2}$. A 
continuación, se llevaron a cabo correlaciones de Spearman (variables categóricas) y de Pearson (variables continuas), con el total de la muestra, para analizar las asociaciones entre características sociodemográficas, clínicas, procesos psicológicos y la toma de decisión compartida. A través de un MANCOVA, en el que se controlaron la edad, el nivel educativo y el tiempo de tratamiento, ya que habían mostrado previamente estar correlacionadas con la preferencia en la toma de decisión, se identificaron diferencias en procesos psicológicos entre los participantes según su preferencia en la participación en la toma de decisión como activos, colaborativos o pasivos. Teniendo en cuenta que había una mayor proporción de mujeres que de hombres diagnosticados con trastorno depresivo, se aplicó un nuevo MANCOVA, controlando edad, nivel educativo y tiempo de tratamiento, para analizar las diferencias de género en los procesos psicológicos y en la toma de decisión compartida. Por último, se llevó a cabo un análisis de regresión jerárquica, con el método paso a paso, en el que se tomó como criterio la preferencia en la participación en la toma de decisión, considerándola como variable continua (a mayor puntuación mayor preferencia por un estilo pasivo). Se controlaron, en el primer paso, la edad y el nivel educativo; en el segundo paso, el tiempo de tratamiento, y en el tercer paso se incluyeron, como variables predictoras, las medidas de procesos psicológicos (locus de control, reactancia y autoeficacia) para cada género por separado.

\section{RESULTADOS}

En este apartado se presentan los resultados de los análisis de correlación entre las características sociodemográficas, clínicas y de procesos psicológicos con la toma de decisió compartida. Seguidamente, se exponen los resultados relativos a las variables clínicas y los procesos psicológicos que están en la base de la toma de decisión compartida, considerando el género como una variable moduladora.

Relación de las características sociodemográficas y clínicas con la toma de decisión compartida

Los análisis preliminares donde se compararon los pacientes diagnosticados con episodio depresivo con los de trastorno depresivo recurrente mostraron diferencias en edad $\left(\chi^{2}(63)=88.23, p<.05\right)$ y género $\left(\chi^{2}(1)=17.98, p<.001\right)$. Los pacientes de mayor edad presentaron más depresión recurrente y los hombres mostraron más depresión aguda que recurrente (no se encontraron diferencias entre las mujeres en el tipo de depresión). Sin embargo, no aparecieron diferencias significativas en nivel educativo $\left(\chi^{2}(3)=6.43\right.$, $p=.093)$, ni en la variable de interés, la preferencia por la toma de decisión compartida $\left(\chi^{2}(5)=4.83, p=.436\right)$. Un MANOVA entre pacientes con depresión aguda y recurrente tampoco mostró diferencias significativas entre los grupos en ninguno de los procesos psicológicos. De ahí que para el resto de análisis se tomó la muestra en conjunto, sin hacer distinción entre depresión aguda y recurrente.

Los resultados mostraron que la preferencia en la toma de decisión se relacionaba con la edad $(r=.28, p<.001)$ y el nivel educativo $(r=-.17, p<.001)$. Las personas de mayor edad preferían una toma de decisión más pasiva, mientras que las de mayor nivel de estudios se mostraban más activas. Además, las personas con preferencia pasiva en la toma de decisión llevaban más tiempo en tratamiento psiquiátrico $(r=.09, p<.05)$. También se encontró que la preferencia pasiva en la toma de decisión se relacionó con mayor locus centrado en el psiquiatra $(r=.16, p<.001)$ o en el azar $(r$ $=.11, p<.05) \mathrm{y}$ con menor autoeficacia $(r=-.10, p<.05)$. Relación entre toma de decisión compartida y procesos psicológicos

Tabla 2.

Contraste en procesos psicológicos en función de la preferencia en toma de decisiones, controlando la edad, el nivel educativo y el tiempo de tratamiento

\begin{tabular}{|c|c|c|c|c|c|}
\hline & $\begin{array}{l}\text { Activo } \\
\mathrm{N}=21 \\
\mathrm{M} \text { D.T. }\end{array}$ & $\begin{array}{c}\text { Colaborativo } \\
\mathrm{N}=304 \\
\text { M D.T. }\end{array}$ & $\begin{array}{l}\text { Pasivo } \\
\mathrm{N}=137 \\
\text { M D.T. }\end{array}$ & $\mathrm{F}$ & $\eta 2$ \\
\hline Locus-interno & 4.421 .79 & 3.911 .21 & 3.941 .21 & 1.82 & \\
\hline Locus-médico & 4.351 .56 & 5.021 .10 & 5.171 .01 & $4.64 * *$ & \\
\hline Locus-azar & 2.05 .83 & 2.421 .17 & 2.591 .17 & 1.99 & \\
\hline Locus-otros & 3.521 .31 & 3.741 .30 & 3.671 .17 & .52 & .02 \\
\hline Reactancia afectiva & 3.231 .32 & 3.451 .08 & 3.191 .16 & 1.01 & \\
\hline Reactancia cognitiva & 2.211 .09 & 2.03 .75 & 1.95 .82 & .63 & \\
\hline Autoeficacia & 3.03 .54 & 2.88 .68 & 2.78 .70 & .78 & \\
\hline
\end{tabular}

Nota: $p<.05^{*}, p<.01^{* *}, p<.001^{* * *}$ 
En la tabla 2 se muestran los datos obtenidos en el MANCOVA. Los resultados mostraron diferencias significativas entre los tres grupos de preferencia en la toma de decisión únicamente en locus centrado en el psiquiatra $(F(2,455)=4.64, p<.01, \eta 2=.02)$. Tras el análisis post-hoc de Bonferroni se encontró que presentaban mayor locus de control centrado en el psiquiatra las personas colaboradoras y pasivas frente a las activas.

Los resultados del MANCOVA tomando el género como variable independiente mostraron que los hombres tenían un mayor locus de control interno $(F(1,456)=5.08, p<.05, \eta 2$ $=.01) \mathrm{y}$ mayor reactancia cognitiva $(F(1,456)=3.64, p=.06$, $\eta 2=.008)$ que las mujeres, mientras que ellas presentaban mayor locus externo centrado en el azar $(F(1,456)=3.09$, $p=.08, \eta 2=.007$ ), aunque las diferencias en reactancia y locus-azar sólo alcanzaron significación estadística marginal. No se encontraron diferencias significativas entre hombres y mujeres en la participación preferida en la toma de decisión.

Por último, en la Tabla 3 se muestra un resumen de los análisis de regresión. Los resultados indicaron que para los hombres solo el locus de control centrado en el psiquiatra predecía la toma de decisión pasiva. Sin embargo, en el caso de las mujeres, la edad explicaba un $9 \%$ de la varianza, cuando entraba en primer lugar, y el locus de control centrado en la confianza en su psiquiatra, el locus de control externo basado en el azar y la escasa autoeficacia explicaban alrededor de un 3\% adicional de la preferencia pasiva por la toma de decisión.

Tabla 3.

Análisis de regresión con las características sociodemográficas, clínicas y procesos psicológicos como predictoras y la preferencia en toma de decisiones como criterio

\begin{tabular}{|c|c|c|c|c|c|c|c|c|}
\hline & \multicolumn{4}{|c|}{ Hombres } & \multicolumn{4}{|c|}{ Mujeres } \\
\hline Variable & $R^{2}$ adj & $\Delta R$ & $\beta$ & $F$ & $R^{2}$ adj & $\Delta R$ & $\beta$ & $F$ \\
\hline Paso 1: & .05 & .06 & & $6.52 *$ & & & & \\
\hline Locus- médico & & & $.25^{*}$ & & & & & \\
\hline Paso 1: & & & & & .09 & .09 & & $35.31 * * *$ \\
\hline Edad & & & & & & & $.30^{* * *}$ & \\
\hline Paso 2: & & & & & .10 & .01 & & $20.80 * * *$ \\
\hline Edad & & & & & & & $.30^{* * *}$ & \\
\hline Locus-azar & & & & & & & $.12 *$ & \\
\hline Paso 3: & & & & & .11 & .01 & $29 * * *$ & $15.61 * * *$ \\
\hline Edad & & & & & & & $.12 *$ & \\
\hline $\begin{array}{l}\text { Locus-azar } \\
\text { Locus-médico }\end{array}$ & & & & & & & $.11 *$ & \\
\hline Paso 4: & & & & & .12 & .01 & $.28 * * *$ & $13.05^{* * *}$ \\
\hline $\begin{array}{l}\text { Edad } \\
\text { Locus-azar }\end{array}$ & & & & & & & $.11^{*}$ & \\
\hline $\begin{array}{l}\text { Locus-médico } \\
\text { Autoeficacia }\end{array}$ & & & & & & & $\begin{array}{l}.11^{*} \\
-.11 *\end{array}$ & \\
\hline
\end{tabular}

Nota: $p<.05^{*}, p<.01^{* *}, p<.001^{* * *}$ 
Los resultados obtenidos indican que los aspectos relacionados con variables demográficas son significativos. Además, los procesos psicológicos asociados con el carácter pasivo o activo en la toma de decisión compartida han resultado de especial importancia en personas con depresión.

\section{DISCUSIÓN}

El objetivo de este trabajo fue analizar el papel de los procesos psicológicos que pueden estar en la base de las preferencias de los pacientes depresivos en la toma de decisiones médicas, examinando la capacidad predictiva de estos procesos frente a las características sociodemográficas y clínicas.

La mayoría de los participantes adoptaban un rol pasivocolaborativo o completamente pasivo en relación con la toma de decisión sobre su tratamiento, tal y como señalan los resultados encontrados en trabajos similares (Arora \& McHorney, 2000; Delgado et al., 2010). Aunque en pacientes con depresión y ansiedad, algunos autores han informado de tasas de hasta el $98 \%$ de participación con un papel semi-activo o semi-pasivo (Patel \& Bakken, 2010). Por otro lado, en este estudio se contaba con una proporción similar de pacientes con depresión aguda $\mathrm{y}$ recurrente, y dicha cronicidad en el diagnostico no afectó la preferencia por la toma de decisión compartida. Sin embargo, se observaron diferencias significativas en edad y género, con mayor depresión recurrente en los pacientes de mayor edad y mayor presencia de depresión aguda que recurrente en los hombres.

Las características sociodemográficas y clínicas que se relacionaron con la preferencia en la toma de decisión fueron la edad, el nivel de estudios y el tiempo de tratamiento. Los resultados indicaron que las personas de mayor edad y que llevaban más tiempo en tratamiento psiquiátrico mostraron preferir un rol más pasivo en la toma de decisiones, mientras que las de mayor nivel de estudios preferían ser más activas. En este sentido, diferentes estudios detectan que las personas mayores y las que llevan largos períodos en tratamiento informan de menor tasa de toma de decisión compartida (Proctor, Hasche, Morrow-Howell, Shumway, \& Snell, 2008; Schneider et al., 2006; Solberg et al., 2014).

Cuando se analizaron las diferencias en los procesos psicológicos entre pacientes que preferían distintos estilos de participación en la toma de decisión, se encontró que a mayor preferencia por una toma de decisión pasiva, mayor locus de control centrado en el profesional. Anteriormente, Hashimoto y Fukuhara (2004) explicaron que la preferencia por la toma de decisión estaba relacionada con la creencia acerca del control que tiene el individuo sobre la salud. Sin embargo, los demás procesos psicológicos analizados en el presente estudio, como la reactancia o la autoeficacia, no parecieron tener un efecto directo en un estilo determinado de toma de decisiones compartidas.

Los resultados mostraron que no existían diferencias de género en la preferencia por la toma de decisión. Algunos estudios han sugerido que las mujeres prefieren asumir un rol activo en la toma de decisión (Levinson et al., 2005), mientras que otros no han encontrado diferencias de género en los distintos roles adoptados (Swanson et al., 2007). En nuestro estudio se encontraron diferencias de género en algunos procesos psicológicos: los hombres tenían mayor locus de control interno y reactancia cognitiva que las mujeres, mientras que ellas presentaban un locus externo centrado en el azar.

Cuando se analizaron los procesos psicológicos implicados en la toma de decisión compartida para hombres y mujeres de manera independiente, se encontraron diferencias de género en el peso de las características sociodemográficas frente a los procesos psicológicos en la predicción del estilo preferido de toma de decisión. En los hombres, únicamente el locus de control centrado en el profesional explicó el estilo pasivo. En el caso de las mujeres, la edad tuvo mayor poder predictivo que los procesos psicológicos, el locus centrado en el profesional y en el azar, y baja autoeficacia. Estudios previos han señalado que la autoevaluación y la habilidad general percibida por el individuo pueden constituir las raíces del inicio o mantenimiento de una decisión determinada (Thunholm, 2004). Este tipo de resultados resaltan la necesidad de abordar la toma de decisiones compartida desde una perspectiva diferencial.

Este estudio ha permitido identificar los principales procesos psicológicos que están en la base de la preferencia por una toma de decisión más activa o pasiva por parte de los pacientes depresivos. En este sentido, el locus de control y la autoeficacia han mostrado ser factores fundamentales en el grado de implicación que el paciente depresivo quiere adoptar durante el proceso de comunicación con su médico, $\mathrm{y}$ estos procesos tienen una mayor o menor relevancia en función del género. Los resultados tienen importantes implicaciones clínicas, dado que ambos procesos pueden ser susceptibles de entrenamiento, de modo que los pacientes aprendan habilidades para hacer frente a la enfermedad y desarrollen un locus de control más interno pero que les permita actuar en colaboración con el médico en el abordaje de la misma.

No obstante, hay que tener en cuenta ciertas limitaciones de este estudio. La escala empleada para evaluar la preferencia por la toma de decisión compartida parte de una clasificación general de los pacientes como activos, pasivos 
o colaboradores, pero puede ocurrir que, dependiendo de la etapa de su enfermedad, deseen tener una mayor o menor implicación en la colaboración con el tratamiento (Flynn, Smith, \& Vanness, 2006).

La mayoría de los participantes de este estudio preferían un papel más pasivo que activo, por lo que sería necesario seguir indagando acerca de los procesos psicológicos que fundamentan una toma de decisión activa. Además, se ha empleado una metodología correlacional que no permite establecer relaciones causales entre las variables implicadas en el proceso de toma de decisión. Por otra parte, la disposición de los pacientes para participar en la toma de decisión también podría estar modulada por la información disponible acerca de su enfermedad, así como por los mitos o creencias erróneas que existen sobre la misma entre la población general, y que llevan al paciente psiquiátrico a sentirse incapacitado para tomar decisiones.

Uno de los desafíos futuros sería lograr una mayor colaboración entre médicos y pacientes en la toma de decisión. El entrenamiento al personal médico en la toma de decisiones compartidas, así como la iniciativa de los pacientes de solicitar información sobre su enfermedad podrían redundar, no solo en una adecuada comunicación médico-paciente, sino además en una mejor adherencia al tratamiento y mayor satisfacción con la atención especializada (Young, Bell, Epstein, Feldman, \& Kravitz, 2008).

\section{REFERENCIAS}

Aljumah, K. \& Hassali, M. A. (2015). Impact of pharmacist intervention on adherence and measurable patient outcomes among depressed patients: A randomised controlled study. BMC Psychiatry, 15, 219-227. http://dx.doi. org/10.1186/s12888-015-0605-8

Arora, N. K. \& McHorney, C. A. (2000). Patient preferences for medical decision making: Who really wants to participate? Medical Care, 38, 335-341. http://dx.doi. org/10.1097/00005650-200003000-00010

Bandura, A. (1977). Self-efficacy: Toward a unifying theory of behavioral change. Psychological Review, 84, 191-215. http://dx.doi.org/10.1037/0033-295X.84.2.191

Botero, P. A. y Londoño, C. (2013). Factores psicosociales predictores de la calidad de vida en personas en situación de discapacidad física. Acta Colombiana de Psicología, 16, 125-137. Retrieved from http://www.scielo.org.co/ scielo.php?pid=S0123-91552013000200012\&script $=$ sci_ arttext\&tlng $=\mathrm{pt}$

Brehm, S. S. \& Brehm, J. W. (2013). Psychological reactance: $A$ theory of freedom and control. New York: Academic Press, INC.
Cantrell, C. R., Eaddy, M. T., Shah, M. B., Regan, T. S. \& Sokol, M. C. (2006). Methods for evaluating patient adherence to antidepressant therapy: A real-world comparison of adherence and economic outcomes. Medical Care, 44, 300-303. http://dx.doi.org/10.1097/01.mlr.0000204287.82701.9b

De Almeida, A. C. \& Chen, T. F. (2008). When pharmacotherapeutic recommendations may lead to the reverse effect on physician decision-making. Pharmacy World \& Science, 30, 3-8. http://dx.doi.org/10.1007/s11096-007-9143-x

De las Cuevas, C. \& Peñate, W. (2014). To what extent psychiatric patients feel involved in decision making about their mental health care? Relationships with socio-demographic, clinical, and psychological variables. Acta Neuropsychiatrica, 26, 372-381. http://dx.doi.org/10.1017/neu.2014.21

De las Cuevas, C., Peñate, W., Betancort, M. \& de Rivera, L. (2014). Psychological reactance in psychiatric patients: Examining the dimensionality and correlates of the Hong Psychological Reactance Scale in a large clinical sample. Personality and Individual Differences, 70, 85-91. http://dx.doi. org/10.1016/j.paid.2014.06.027

De las Cuevas, C., Peñate, W. \& Sanz, E. J. (2014). Risk factors for non-adherence to antidepressant treatment in patients with mood disorders. European Journal of Clinical Pharmacology, 70, 89-98. http://dx.doi.org/10.1007/s00228013-1582-9

De las Cuevas, C., Peñate, W., Betancort, M. \& Cabrera, C. (2015). What Do Psychiatric Patients Believe Regarding Where Control Over Their Illness Lies?: Validation of the Multidimensional Health Locus of Control Scale in Psychiatric Outpatient Care. The Journal of Nervous and Mental Disease, 203(2), 81-86. http://dx.doi.org/10.1097/ NMD.0000000000000244

Degner, L. F., Sloan, J. A. \& Venkatesh, P. (1997). The Control Preferences Scale. Canadian Journal Nursing Research, 29, 21-43.

Delgado, A., López, L. A., Dios, J. D., Saletti, L., Gil, N. \& Puga, A. (2010). Expectativas de los pacientes sobre la toma de decisiones ante diferentes problemas de salud. Gaceta Sanitaria, 24, 66-71. Retrieved from http://www.scielosp.org/ scielo.php?pid=S0213-91112010000100011\&script $=$ sci_ arttext\&tlng=es

Flynn, K. E., Smith, M. A. \& Vanness, D. (2006). A typology of preferences for participation in healthcare decision making. Social Science \& Medicine, 63, 1158-1169. http:// dx.doi.org/10.1016/j.socscimed.2006.03.030

Geddes, J. R., Carney, S. M., Davies, C., Furukawa, T. A., Kupfer, D. J., Frank, E. \& Goodwin, G. M. (2003). Relapse prevention with antidepressant drug treatment in depressive disorders: A systematic review. The Lancet, 361, 653-661. http://dx.doi.org/10.1016/S0140-6736(03)12599-8

Gili, M., Roca, M., Basu, S., McKee, M. \& Stuckler, D. (2013). The mental health risks of economic crisis in Spain: Evidence from primary care centers, 2006 and 2010. The Euro- 
pean Journal of Public Health, 23, 103-108. http://dx.doi. org/10.1093/eurpub/cks035

Grenard, J. L., Munjas, B. A., Adams, J. L., Suttorp, M., Maglione, M., McGlynn, E. A. \& Gellad, W. F. (2011). Depression and medication adherence in the treatment of chronic diseases in the United States: A meta-analysis. Journal of General Internal Medicine, 26, 1175-1182. http://dx.doi.org/10.1007/s11606-011-1704-y

Hashimoto, H. \& Fukuhara, S. (2004). The influence of locus of control on preferences for information and decision making. Patient Education and Counseling, 55, 236-240. http:// dx.doi.org/10.1016/j.pec.2003.09.010

Hong, S. M. \& Faedda, S. (1996). Refinement of the Hong psychological reactance scale. Educational and Psychological Measurement, 56, 173-182. http://dx.doi. org/10.1177/0013164496056001014

Jerusalem, M. \& Schwarzer, R. (1992). Self-efficacy as a resource factor in stress appraisal processes. En R. Schwarzer (Ed.), Self-efficacy: Thought control of action (pp. 195213). Washington, DC: Hemisphere.

Joosten, E. A., De Fuentes-Merillas, L., De Weert, G. H., Sensky, T., Van Der Staak, C. P. F. \& de Jong, C. A. (2008). Systematic review of the effects of shared decision-making on patient satisfaction, treatment adherence and health status. Psychotherapy and Psychosomatics, 77, 219-226. http://dx.doi.org/10.1159/000126073

Katon, W. J. (2011). Epidemiology and treatment of depression in patients with chronic medical illness. Dialogues in Clinical Neuroscience, 13, 7-23. Retrieved from http://www. ncbi.nlm.nih.gov/pmc/articles/PMC3181964/

Levinson, W., Kao, A., Kuby, A. \& Thisted, R. A. (2005). Not all patients want to participate in decision making. A national study of public preferences. Journal of General Internal Medicine, 20, 531-535. http://dx.doi.org/10.1111/j.15251497.2005.04101.x

Loh, A., Simon, D., Wills, C. E., Kriston, L., Niebling, W. \& Härter, M. (2007). The effects of a shared decision-making intervention in primary care of depression: A cluster-randomized controlled trial. Patient Education and Counseling, 67, 324-332. http://dx.doi.org/10.1016/j.pec.2007.03.023

Longtin, Y., Sax, H., Leape, L. L., Sheridan, S. E., Donaldson, L. \& Pittet, D. (2010). Patient participation: Current knowledge and applicability to patient safety. Mayo Clinic Proceedings, 85, 53-62. http://dx.doi.org/10.4065/ mcp.2009.0248

Makoul, G. \& Clayman, M.L. (2006) An integrative model of shared decision making in medical encounters. Patient Education and Counseling, 60, 301-312. http://dx.doi. org/10.1016/j.pec.2005.06.010

O’Hea, E. L., Grothe, K. B., Bodenlos, J. S., Boudreaux, E. D., White, M. A. \& Brantley, P. J. (2005). Predicting medical regimen adherence: The interactions of health locus of control beliefs. Journal of Health Psychology, 10, 705-717. http://dx.doi.org/10.1177/1359105305055330
Patel, S. R. \& Bakken, S. (2010). Preferences for participation in decision making among ethnically diverse patients with anxiety and depression. Community Mental Health Journal, 46, 466-473. http://dx.doi.org/10.1007/s10597-010-9323-3

Pérez-Wehbe, A. I., Perestelo, L., Bethencourt, J. M., Cuéllar, L. $\&$ Peñate, W. (2014). Treatment-resistant depression: A systematic review of systematic reviews. InternationalJournalof Clinical and Health Psychology, 14,145-153. Retrieved from http://apps.elsevier.es/watermark/ctl_servlet?_f $=10 \&$ pident_articulo $=90295524 \&$ pident usuario $=0 \&$ pcontactid $=\&$ pident_revista $=355 \&$ ty $=0 \&$ accio $\mathrm{n}=\mathrm{L} \&$ origen $=$ zonadelectura\&web $=$ zl.elsevier.es\&lan $=\mathrm{en} \&$ fichero $=355 \mathrm{v} 14 \mathrm{n} 02 \mathrm{a} 90295524 \mathrm{pdf001}$.pdf

Proctor, E. K., Hasche, L., Morrow-Howell, N., Shumway, M. \& Snell, G. (2008). Perceptions about competing psychosocial problems and treatment priorities among older adults with depression. Psychiatry Services, 59, 670-5. http:// dx.doi.org/10.1176/ps.2008.59.6.670

Pudrovska, T. (2015). Gender and Health Control Beliefs Among Middle-Aged and Older Adults. Journal of Aging and Health, 27, 284-303. http://dx.doi. org/10.1177/0898264314549659

Sanjuán, P., Pérez, A. M. y Bermúdez, J. (2000). Escala de autoeficacia general: datos psicométricos de la adaptación para población española. Psicothema, 12, 509-513. Retrieved from http://www.unioviedo.es/reunido/index.php/ PST/article/view/7741

Schneider, A., Körner, T., Mehring, M., Wensing, M., Elwyn, G. \& Szecsenyi, J. (2006). Impact of age, health locus of control and psychological co-morbidity on patients' preferences for shared decision making in general practice. $\mathrm{Pa}$ tient Education and Counseling, 61, 292-298. http://dx.doi. org/10.1016/j.pec.2005.04.008

Schwarzer, R. \& Fuchs, R. (1996). Self-efficacy and health behaviours. Predicting health behaviour: Research and practice with social cognition models. Buckingham: Open University Press.

Serra-Taylor, J. y Irizarry-Robles, C.Y. (2015). Factores protectores de la depresión en una muestra de adultos mayores en Puerto Rico: Autoeficacia, escolaridad y otras variables sociodemográficas. Acta Colombiana de Psicología, 18, 125134. http://dx.doi.org/10.14718/ACP.2015.18.1.12

Singh, J. A., Sloan, J. A., Atherton, P. J., Smith, T., Hack, T. F., Huschka, M. M., ... \& Degner, L. F. (2010). Preferred roles in treatment decision making among patients with cancer: A pooled analysis of studies using the Control Preferences Scale. The American Journal of Managed Care, 16, 688696. Retrieved from http://www.ncbi.nlm.nih.gov/pmc/articles/PMC3020073/

Solberg, L. I., Crain, A. L., Rubenstein, L., Unützer, J., Whitebird, R. R. \& Beck, A. (2014). How much shared decision making occurs in usual primary care of depression? The Journal of the American Board of Family Medicine, 27, 199-208. http://dx.doi.org/10.3122/ jabfm.2014.02.130164 
Swanson, K. A., Bastani, R., Rubenstein, L. V., Meredith, L. S. \& Ford, D. E. (2007). Effect of mental health care and shared decision making on patient satisfaction in a community sample of patients with depression. Medical Care Research and Review, 64, 416-430. http://dx.doi. org/10.1177/1077558707299479

Tariman, J. D., Doorenbos, A., Schepp, K. G., Singhal, S. \& Berry, D. L. (2014). Older adults newly diagnosed with symptomatic myeloma and treatment decision making. Oncology Nursing Forum, 41, 411-419. http://dx.doi.org/10.1188/14

Thunholm, P. (2004). Decision-making style: Habit, style or both? Personality and Individual Differences, 36, 931-944. http://dx.doi.org/10.1016/S0191-8869(03)00162-4

Wallston, K. A. (1992). Hocus-pocus, the focus isn't strictly on locus: Rotter's social learning theory modified for health.
Cognitive Therapy and Research, 16, 183-199. http:// dx.doi.org/10.1007/BF01173488

Wallston, K. A., Stein, M. J. \& Smith, C. A. (1994). Form C of the MHLC scales: A condition-specific measure of locus of control. Journal of Personality Assessment, 63, 534-553. http://dx.doi.org/10.1207/s15327752jpa6303_10

World Health Organization. (2004). International statistical classification of diseases and health related problems (The) ICD-10. World Health Organization.

Young, H. N., Bell, R. A., Epstein, R. M., Feldman, M. D. \& Kravitz, R. L. (2008). Physicians' shared decision-making behaviors in depression care. Archives of Internal Medicine, 168, 1404-1408. http://dx.doi.org/10.1001/archinte.168.13.1404 